\title{
Identification of Y118 Amino Acid Residue in Candida albicans Sterol $14 \alpha$-Demethylase Associated with the Enzyme Activity and Selective Antifungal Activity of Azole Analogues
}

\author{
Shuang-Hong Chen, ${ }^{b}$ Chun-Quan SHENG, ${ }^{a}$ Xiao-Hui Xu, ${ }^{c}$ Yuan-Ying JIAng, ${ }^{* a}$ \\ Wan-Nian ZHANG, ${ }^{*}, a$ and Cheng $\mathrm{HE}^{c}$ \\ ${ }^{a}$ School of Pharmacy, Second Military Medical University; 325 Guohe Road, Shanghai 200433, P.R. China: ${ }^{b}$ Naval \\ Medical Research Institute; 880 Xiangying Road, Shanghai 200433, P.R. China: and ${ }^{c}$ Department of Neurobiology, \\ Second Military Medical University; 800 Xiangying Road, Shanghai 200433, P.R. China. \\ Received February 25, 2007; accepted April 16, 2007
}

\begin{abstract}
Our previous publication established a model to predict that the phenyl group of the $\mathrm{C}-3$ side chain of azole antifungal compounds interacts with the phenol group of Tyr118 through the formation of $\pi$ - $\pi$ face-to-edge interaction. To verify this prediction, wild type and three site-directed mutants of the $Y 118$ residue of Candida albicans sterol 14 $\alpha$-demethylase P450 (CACYP51) were constructed and heterologously expressed in Saccharomyces cerevisiae with deletion of the CYP51 gene. With the strains obtained and microsome enzymes separated, cell susceptibility and CACYP51 activity were examined with the 5 novel azole compounds based on the molecular modeling in comparison with fluconazole. After alteration of $Y 118$ with $Y 118 A, Y 118 F$, and $Y 118 T$ by a single base substitution, the expression levels of CACYP51 protein were not affected. However, these mutations markedly decreased its catalytic activity respectively; the mutation changes also decreased azole susceptibility, indicating the structural importance of the Y118 residue in maintaining CACYP51 activity and in determining azole susceptibility. In addition, our synthetic compounds with the phenyl group side chain attached to $\mathrm{C} 3$ produced higher susceptibility against $S$. cerevisiae with expression of CACYP51 and exhibited more potent inhibitory effects on CACYP51 activity in comparison with fluconazole, suggesting that the phenyl group of C3 side chain substitutes is also important for selective binding to target enzymes.
\end{abstract}

Key words Candida albicans; gene deletion; mutation; inhibitor binding; CYP51

Azoles are widely used in the treatment of fungal infections. They act by competitive inhibition of lanosterol $14 \alpha$ demethylase (P45014DM, CYP51), the key enzyme in sterol biosynthesis, which results in depletion of ergosterol and accumulation of lanosterol and other 14-methyl sterols ultimately leading to the growth inhibition of fungal cells. ${ }^{1)}$ Inhibition of the ergosterol biosynthetic pathway in fungi has thus attracted intense interests in the application of such antifungal compounds. However, it was found in the past two decades that azole resistance often develops prolonged use of oral imidazole and triazole, or different forms of immunodeficiency in fungal infection patients. ${ }^{2)}$ Furthermore, cross inhibition of CYP51 in different species also causes undesirable side effects. ${ }^{3)}$ It is, therefore, of increasingly interest to develop novel antifungal agents with more selective antifungal activity, higher efficiency, broader pectrum, and lower toxicity.

Azole antifungal agents inhibit the CYP51 by a mechanism in which the heterocyclic nitrogen atom (N-3 of imidazole and $\mathrm{N}-4$ of triazole) binds to the heme iron atom in the binding site of the enzyme. Because all the fungal CYP51 proteins that had been characterized were membrane-bound and difficult to solve their crystal structures, the study of the interaction between azoles and fungal CYP51 can only be done by methods of molecular modeling. Several three-dimensional (3D) models of CYP51 and their interaction with azole antifungal agents has been reported. Ji et al. ${ }^{3,4)}$ built a homologous 3D model of CYP51 from C. albicans based on the crystal coordinates of all four known prokaryotic P450s. With this model they found that the halogenated phenyl group of azole inhibitors is deep in the hydrophobic binding cleft and the long side chains of some inhibitors such as itra- conazole and ketoconazole surpass the active site and interact with the residues in the substrate access channel. Another 3D molecular model constructed by Lewis et al. ${ }^{5)}$ also showed that typical azole inhibitors were able to fit the putative active site of CYP51 by a combination of heme coordination, hydrogen bonding, $\pi-\pi$ stacking and hydrophobic interactions within the heme environment of the enzymes. Recently, modelling data of $\mathrm{Li}$ et al. $^{6}{ }^{6}$ suggest that the long chain of posaconazole and itraconazole occupies a specific channel within CYP51 and that this additional interaction serves to stabilize the binding of these azoles to the mutated CYP51 proteins. Models generated by Fukuoka et al. ${ }^{7)}$ predicted that voriconazole was a more potent inhibitor than fluconazole because the additional methyl group of voriconazole resulted in stronger hydrophobic interaction with the aromatic amino acids in the substrate binding site and filled the site more extensively.

In our studies, the 3D model of CYP51 from Candida albicans (CACYP51) was constructed. ${ }^{3)}$ In addition, the binding mode of the substrate with fungal CYP51 was also investigated. ${ }^{3)}$ In order to search more potent azoles antifungal agents and design lead compounds with higher affinity, the binding mode of azoles antifungal agents with CACYP51 was explored using the previously described molecular docking and 3D-QSAR methods. ${ }^{3)}$ And a structure-based pharmacophore model was therefore established to guide the rational optimization of the azole antifungal agents. ${ }^{3,8-10)}$ This model predicted a hypothesis that the phenyl group of the C-3 side chain of azole antifungal compounds interacts with the phenol group of Tyr118, a highly conserved residue in CYP51 family, through the formation of $\pi-\pi$ face-to-edge interaction (Fig. 1). To demonstrate this hypothesis and verify the 


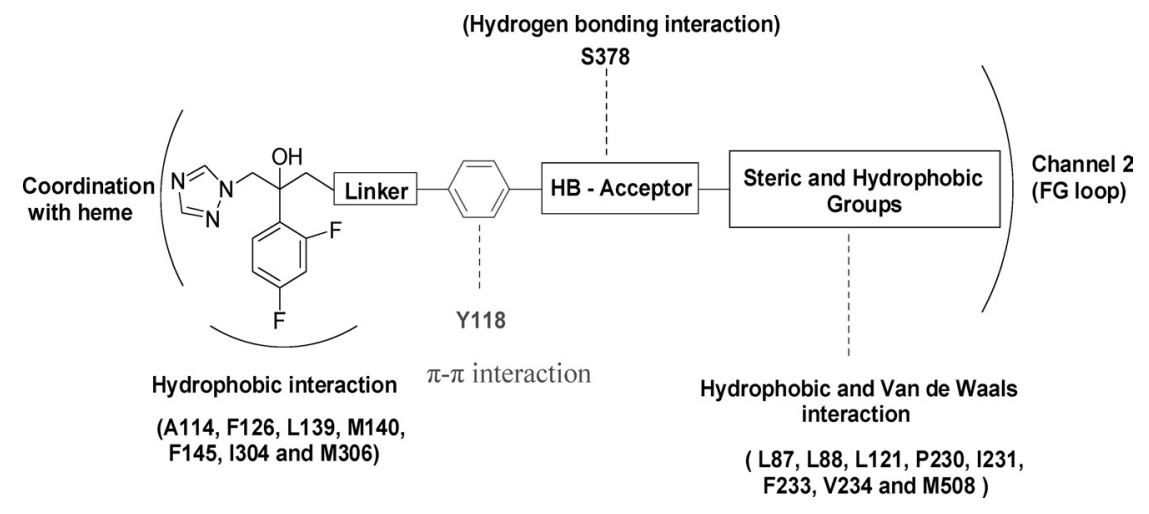

Fig. 1. Interaction Model of Compounds with Y118 Amino Acid

reliability of the derived pharmacophore model, a series of novel azole compounds on the basis of this model were synthesized and tested for their in vitro antifungal activities in our laboratory. One of the major objectives of the present study is to reveal that these azole compounds will have excellent in vitro activities and the structure-activity relationship correlated with the model. As a result, our synthesized compounds may not only be used to perform further biological evaluations to develop new candidates of antifungal drugs, but also can be used as "probes" to investigate the structure-function relationship of CYP51 family.

Up to date, the X-ray crystal structure of CYP51 from $M y$ cobacterium tuberculosis has already been solved, but the explicit information about enzyme binding site of pathogenic fungi is not available yet. Even when the structure of a complex is available, it is still very difficult to deduce the relative contribution of each individual residue to the total binding energy. ${ }^{1-13)}$ Mutational analysis, therefore, remains to be the mainstay of molecular determination of binding interfaces between receptor-ligand complexes. Although there are a number of papers reporting mutation analyses of current azole antifungal agents, they focused more on the mechanism of drug resistance, ${ }^{14-16)}$ and site-directed mutagenesis pinpointing key residues responsible for substrate or inhibitors binding were never reported. ${ }^{4)}$ In the present study, site-directed mutagenesis of the $Y 118$ residue of $C$. albicans CYP51 heterologously expressed in S. cerevisiae was undertaken in an attempt to verify our previous molecular modeling by comparative assessment of the strain susceptibility and enzyme binding to a set of azoles antifungals.

\section{MATERIALS AND METHODS}

Strains Plasmids and Reagents $S$. cerevisiae strains were provided by the Invitrogen (California, American). $C$. albicans collection strain SC5314 was kindly provided by Dr. William A. Fonzi (Department of Microbiology and Immunology, Georgetown University, Washington, U.S.A.). Y12667 (BY4742; Mat $\alpha$; his3,1; leu2,0; lys2,0; ura3,0; YLR056w::kanMX4), the derivations of yeast S288C, was used as deletion parental strains in the study. Yeast strains were routinely grown on yeast peptone dextrose (YPD) rich media. Synthetic complete (SC) media contained $2 \%$ glucose, $1.67 \mathrm{~g} / 1$ yeast nitrogen base without amino acids, and $5 \mathrm{~g} / 1$ ammonium sulfate supplemented with the required nutrients. Plasmids were propagated in $\mathrm{DH} 5 \alpha$ strain. The
pTEF1/Zeo plasmid (from Invitrogen) containing the bacterial Zeocin gene as a selectable marker that confers resistance to Zeocin in yeast was used to construct the disruption cassettes. Plasmids $P Y C / N T / C$ (from Invitrogen) was used for cloning the ERG11 gene from SC5314. The restriction endonucleases, Pryobest DNA polymerase and T4 DNA ligase were purchased from Takara Biotechnology Co., Ltd. Cultural reagents, westeron blot kits and Anti-Xpress (amino acid tag of $P Y C / N T / C$ plasmid) monoclonal antibody were obtained from Invitrogen. PCR site directed mutation kits were from Strategen, Amsterdam, Netherlands. Electroporation cuvette was from Bio-Rad. All other chemicals were purchased from Sigma. Anti-CYP51 antibody was made by our laboratory. All plasmids with detailed maps are deposited in the Invitrogen collection.

Gene Deletion Standard methods for DNA manipulations were referring as described by Brachmann et al. ${ }^{27,28)}$ Briefly, S. cerevisiae S288C genomic DNA was used as the template. $p T E F 1 /$ Zeo was used as the manipulated plasmid. Disruption cassette for the open reading frame (ORF) was constructed according to the following steps. At first, the resultant DNA fragment of up $69 \mathrm{bp}$ oligonucleotides containing $45 \mathrm{bp}$ region homologous to upstream of the coding region of S288C ERG11 gene was ligated into the SacI and $X$ XoI restrict sites of the $p T E F 1 / Z e o$ plasmid $5 \mathrm{k}$ region. The down 69 bp oligonucleotides including 45 bp region homologous to downstream of the stop codon of S288C ERG11 gene was ligated into the ApaI and Pst restrict sites of the $p T E F 1 / Z e o$ plasmid $3 \mathrm{k}$ region. Insert orientation in the recombinant plasmids ( $p T E F 1 / Z e o$ ) was verified with restriction enzymes, and the promoter and terminator regions were confirmed by sequencing. Then, Disruption cassettes were released from recombinant $p T E F 1 / Z e o$ by restriction digestion at the ApaI and PstI sites and transformed to strain Y12667 (Fig. 3). The upstream/downstream 45 bp target homology sequence and the verified primers are deposited in Standford Yeast Deletion homepage (http://www.yeastdeletionhomepage). Table 1 defined all the primers used in the study. Figure 3 defined the map of the manipulated plasmid and the strategy of constructed the deletion cassette. Yeast cells with DNA fragment digested from the recombinant plasmid were transformed to Zeocin resistance $(50 \mu \mathrm{g} / \mathrm{ml})$ and $\mathrm{G} 418$ resistance $(100 \mu \mathrm{g} / \mathrm{ml})$ plate to produce the new strains D12667. Correct targeting of the replacement cassette at the genomic locus was verified by PCR analysis (referring to the standford strain confirmation protocol, http://www. 
Table 1. Sequence of Oligonucleotide Primers Used to Generate Disruption and Mutations

\begin{tabular}{|c|c|}
\hline Name & Primer sequences \\
\hline \multicolumn{2}{|l|}{ For SC5314 ORF ERG11 clone } \\
\hline ERG11-forward 1 & 5'-TGTTGAAACTGTCATTGATGG-3' \\
\hline$E R G 11$-reverse 1 & 5'-CACTGAATCGAAAGAAAGTTGC-3' \\
\hline ERG11-forward 2 & 5'-AAAGGATCCATGGCTATTCTTGAAACTGTCATTGATGGC-3' \\
\hline ERG11-reverse 2 & 5'-AAAGAATTCTTAAAACATACAAGTTTCTC-3' \\
\hline \multicolumn{2}{|c|}{ For Y12667 ORF ERG11 deletion } \\
\hline upstream 45 bp-forward & $\begin{array}{l}\text { 5'-AAAGAGCTCGAATTCCAGCAGGCTTGAATAGAAACAGAACAAACGAGTAATACAAGGATGCTCGA- } \\
\text { GAAA-3' }\end{array}$ \\
\hline upstream 45 bp-reverse & 5'-TTTCTCGAGCATCCTTGTATTACTCGTTTGTTCTGTTTCTATTCAAGCCTGCTGGAATTCGAGCTCTTT-3' \\
\hline downstream 45 bp-forward & $\begin{array}{l}\text { 5'-AAAGGATCCTAAGGTTTGTAACTGAGAAAAAAAAAAAAAACAGAAAAAGAGAGAGGGCCCCTGCA- } \\
\text { GAAA-3' }\end{array}$ \\
\hline downstream 45 bp-reverse & 5'-TTTCTGCAGGGGCCCTCTCTCTTTTTCTGTTTTTTTTTTTTTTCTCAGTTACAAACCTTAGGATCCTTT-3' \\
\hline \multicolumn{2}{|c|}{ For Y12667 ERG11 $\Delta$ verification } \\
\hline A & 5'-CATGTATTCGAAAAGCCTCTAAAAA-3' \\
\hline B & 5'-СТCTTCCTAACAAAACGAATGAAAA-3' \\
\hline $\mathrm{C}$ & 5'-AACAAAGATTCTGCCTCCTCTTATT-3' \\
\hline $\mathrm{D}$ & 5'-ATTGCAAAAAGATTTACAAGTTTGC-3' \\
\hline Zeocin B & 5'-CAACACCGCCCCTTAGATTAG-3' \\
\hline Zeocin $\mathrm{C}$ & 5'-ATCCGTCCCCCTTTTCCTTTG-3' \\
\hline \multicolumn{2}{|l|}{ For $E R G 11$ mutations } \\
\hline Y118A-forward & 5'-TCTGCTGAAGATGCTGCTAAACATTTAACTACT-3' \\
\hline Y118A-reverse & 5'-AGTAGTTAAATGTTTAGCAGCATCTTCAGCAGA-3' \\
\hline Y118F-forward & 5'-TCTGCTGAAGATGCTTTCAAACATTTAACTACT-3' \\
\hline Y118F-reverse & 5'-AGTAGTTAAATGTTTGAAAGCATCTTCAGCAGA-3' \\
\hline Y118T-forward & 5'-TCTGCTGAAGATGCTCAGAAACATTTAACTACT-3' \\
\hline Y118T-reverse & 5'-AGTAGTTAAATGTTTCTGAGCATCTTCAGCAGA-3' \\
\hline
\end{tabular}

yeastdeletionhomepage). Finally, the inactive function of ERG11 was further assessed by cell growth, protein expression and lanosterol composing analysis.

Site Directed Mutation The whole coding region of ERG11 gene was amplified from SC5314 genomic DNA and cloned to vector $P Y C / N T / C$ to produce the recombinant plasmid RePYC/ERG11/WT. Site mutation was employed with site-directed mutation kits according to the manufacture instructions and manipulation methods. ${ }^{14-16,20)}$ PCR reactions were performed on Bio-Rad DNA thermal cycler with conditions consisting of an initial $30 \mathrm{~s}$ denaturation at $95^{\circ} \mathrm{C}$, an annealing step for $30 \mathrm{~s}$ at $55^{\circ} \mathrm{C}$, and an extension step for $20 \mathrm{~min}$ at $68^{\circ} \mathrm{C}$, followed by 18 cycles. PCR was undertaken using $P f u$ polymerase (Stratagene). The resulting DNA fragments containing the $Y 118 A, Y 118 F$ and $Y 118 T$ mutations were ligated into the $P Y C / N T / C$ expression plasmid respectively and annotated as $\operatorname{RePYC/ERG11/YA,RePYC/ERG11/}$ $Y F, R e P Y C / E R G 11 / Y T$. Introduction of the mutation and maintenance of the authentic sequence was corroborated by DNA sequencing (Invitrogene Corp.).

Heterologous Expression of Recombinant Proteins To express the wild type and the mutants Sc5314 CYP51 proteins, the plasmid RePYC/ERG11/WT, RePYC/ERG11/YA,

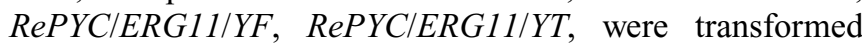
into D12667 to produce the strains Re12667WT, Re12667YA, Re12667YF, Re12667YT, respectively. Yeast transformants were grown at $30^{\circ} \mathrm{C}, 250 \mathrm{rpm}$ with a $1000 \mathrm{ml}$ culture in $2000 \mathrm{ml}$ flasks. The media used consisted of Difco yeast nitrogen base without amino acids $(1.34 \% \mathrm{w} / \mathrm{v})$ supplemented with $100 \mathrm{mg} / \mathrm{l}$ multi amino acids and $2 \%(\mathrm{w} / \mathrm{v})$ glucose as an initial carbon source. Heterologous expression was induced when the glucose was exhausted at a cell density of approximately $10^{8}$ cells $/ \mathrm{ml}$. The culture was left a further $4 \mathrm{~h}$ before galactose was added to a concentration of $2 \%$ $(\mathrm{w} / \mathrm{v})$. After a $20 \mathrm{~h}$ induction, cells were harvested by centrifugation, resuspended in buffer containing $100 \mathrm{~mm}$ potassium phosphate [pH 7.4], $1 \mathrm{~mm}$ nicotinamide, $1 \mathrm{~mm}$ PMSF, $5 \mathrm{~mm}$ glutathione, $10 \%(\mathrm{v} / \mathrm{v})$ glycerol. The cells were mixed with an equal volume of glass beads (diameter, 0.45 to $0.5 \mathrm{~mm}$ ) and were homogenized with a cell homogenizer (Braun $\mathrm{GmbH}$, Mesungen, Germany) operating at $4000 \mathrm{rpm}$ with seven $10 \mathrm{~s}$ bursts and intervals of $30 \mathrm{~s}$ with liquid carbon dioxide cooling. Cell debris was removed by centrifugation at $2000 \boldsymbol{g}$ for $5 \mathrm{~min}$. The resulting supernatant was centrifuged twice at $10000 \mathrm{~g}$ for $20 \mathrm{~min}$ to remove mitochondria and then at $100000 \mathrm{~g}$ for $120 \mathrm{~min}$ to yield the microsome pellet. The pellet was resuspended in buffer containing $100 \mathrm{~mm}$ potassium phosphate [pH 7.4], $1 \mathrm{~mm}$ nicotinamide, $5 \mathrm{~mm}$ glutathione, $20 \%(\mathrm{v} / \mathrm{v})$ glycerol and stored at $-80{ }^{\circ} \mathrm{C}$ before use. The protein concentration was quantified by the method of Sanglard et al. ${ }^{14-18,20)}$

Determination of Sterol 14 $\alpha$-Demethylase Activity of the Microsomes CYP51 activity was determined with a reconstituted system consisting of microsome CYP51. The reaction mixture was incubated at $30^{\circ} \mathrm{C}$ for $2 \mathrm{~min}$, which contained a solution of nicotinamide adenine dinucleotide phosphate, oxidized $\left(\mathrm{NADP}^{+}, 2 \mathrm{mg}\right)$, glucose 6-phosphate $(4 \mathrm{mg})$ and glucose-6-phosphate dehydrogenase (4 units) in a $100 \mathrm{~mm}$ potassium phosphate buffer with $1 \mathrm{~mm}$ ethylenediaminetetraacetic acid (EDTA), $1 \mathrm{~mm}$ glutathione, and $20 \%$ $(\mathrm{v} / \mathrm{v})$ glycerol $(0.5 \mathrm{ml}, \mathrm{pH} 7.4)$. To this mixture was added microsomal protein approximately $10 \mathrm{mg}$, and the volume was made up to $1 \mathrm{ml}$ with the above buffer. Following the addition of the $32.4 \mathrm{nmol}$ lanosterol dispersed with dilauroylphosphatidyl choline..$^{2,20-24)}$ The reaction was run aerobically at $30^{\circ} \mathrm{C}$ for another $25 \mathrm{~min}$ with shaking (100 rpm). The reaction was then stopped by adding $1 \mathrm{ml}$ of freshly prepared saponification reagent $(10 \%[\mathrm{w} / \mathrm{v}] \mathrm{KOH}$ in $90 \%[\mathrm{v} / \mathrm{v}]$ 
ethanol); the mixture was heated at $80^{\circ} \mathrm{C}$ for $1 \mathrm{~h}$ for saponification. sterols were extracted twice with $2 \mathrm{ml}$ of $n$-heptane and were dried under nitrogen at $40{ }^{\circ} \mathrm{C}$. Sterols extracted from the reaction mixture were then analyzed by gas chromatography mass spectroscopy (GC-MS), ${ }^{29)}$ and CYP51 activity was calculated from the conversion ratio of the substrate to the 14-demethylated metabolites as described previously. ${ }^{2)}$ The above procedure repeated twice.

Inhibition of Microsome Sterol $14 \alpha$-Demethylase Activity Sterol $14 \alpha$-demethylase activity of the preparations of microsome ReCYP51WT, ReCYP51YA, ReCYP51YF, ReCYP51YT was assayed as described above. Azole antifungal, fluconazole, idioconazole, A3 and E8 (Fig. 2), were added to the reaction mixtures with the concentration range from 0 to $0.8 \mu \mathrm{M}$, respectively, from 1000 -fold stock solutions. Sterols extracted from the reaction mixture were analyzed by GC-MS. ${ }^{29)}$

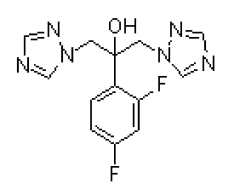

Fluconazole

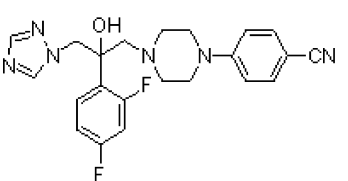

A3

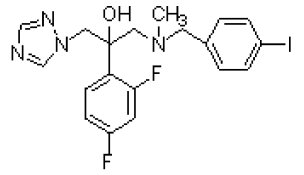

odiconazole
Fig. 2. Structures of Azole Compounds Used in This Study

$\mathbf{A}$

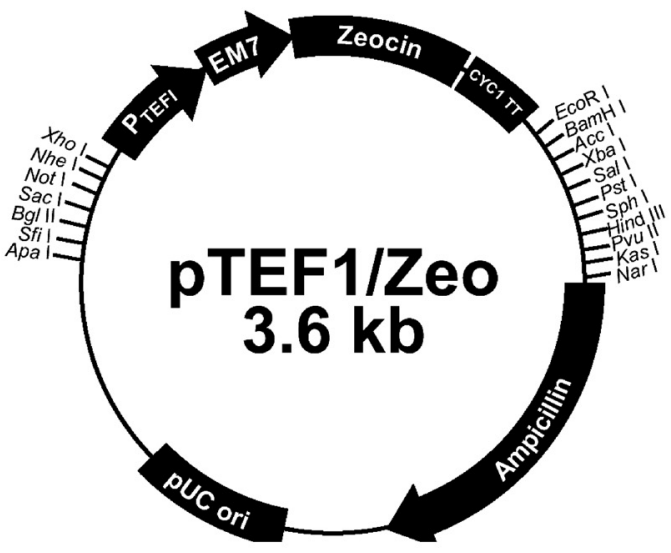

B

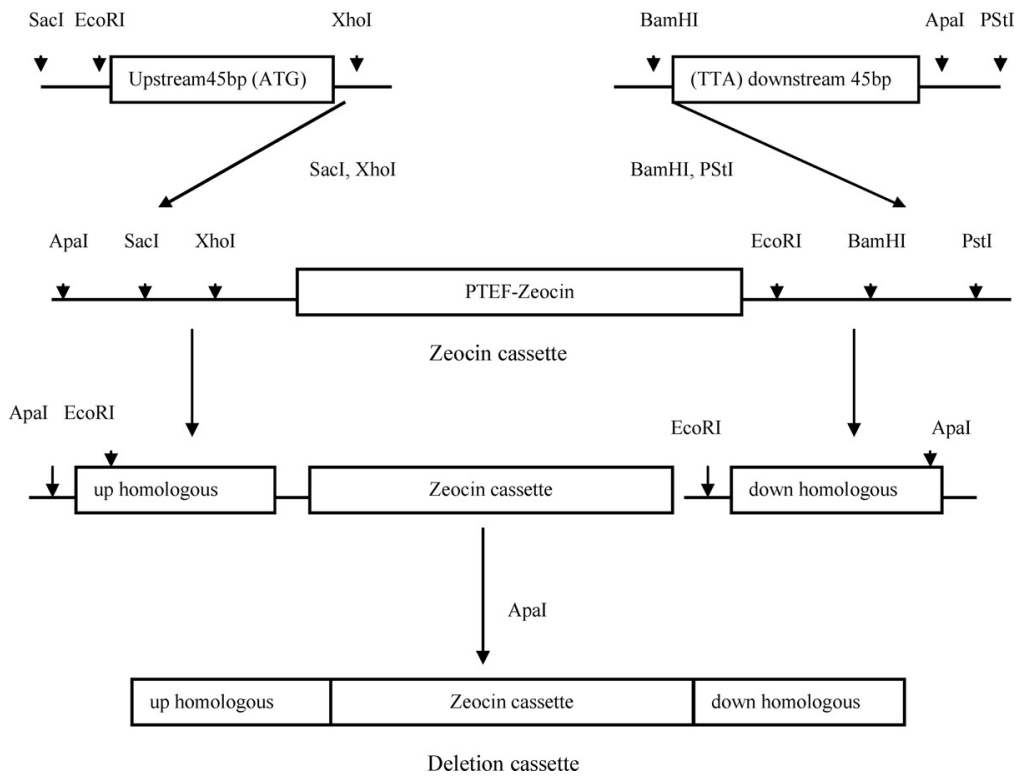

Fig. 3. Map of Manipulated Plasmids and Strategy to Construct the Deletion Cassette

(A) pTEF $1 /$ Zeocin. (B) Construction the deletion cassette. 


\section{RESULTS AND DISCUSSION}

Deletion of the ERG11 Gene and Heterologous Expression of CYP51 Wild Type and Variants Previous studies $^{5,18,29)}$ have demonstrated that endogenous CYP51 protein in parental $S$. cerevisiae interferes with exogenous CYP51 protein expression and functional analysis in the cell-based or microsome based level without purification. ERG11 is an essential requirement gene for yeast viability, ${ }^{5,18,29)}$ and $S$. cerevisiae strains with lethal mutations in the ERG11 gene survive only in an anaerobic environment and require medium supplemented with ergosta-5,7,22-trien-3 $\beta$-ol (er-
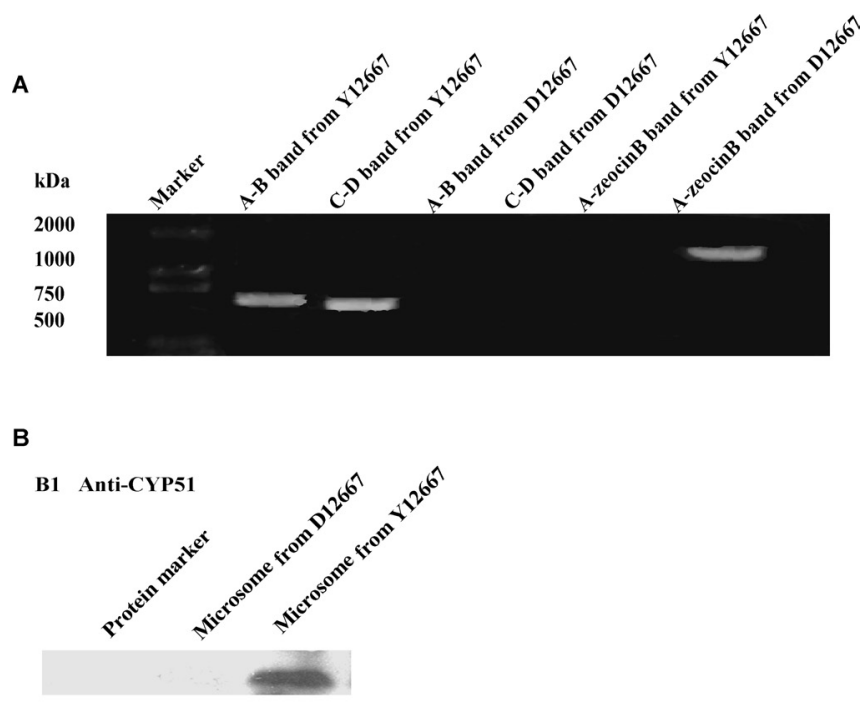

B2 Anti Xpress

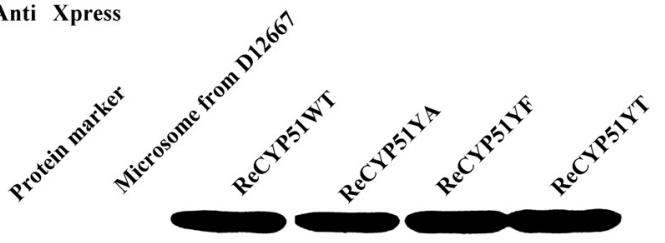

C

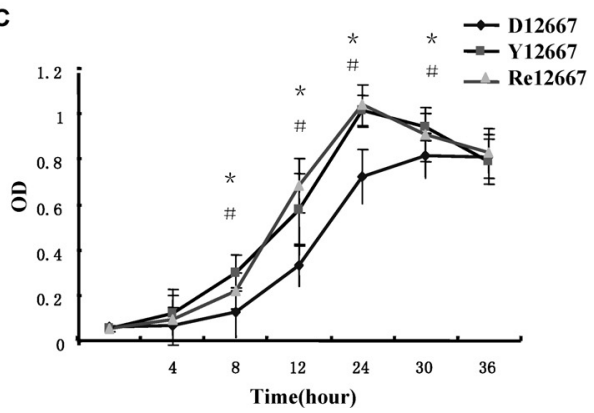

Fig. 4. Disruption, Expression and Growth of ERG11 and Mutants in Yeast

(A) PCR analysis of the ERG11 deletion in Y12667. The "A" and "D" primers were positioned $200-400 \mathrm{bp}$ from the start and stop codons of the Y12667ERG11 gene, respectively. The "zeocinB" "zeocinC" primers are internal to the zeocin module. For a successful deletion case, $\mathrm{A} / \mathrm{B}$ band and $\mathrm{C} / \mathrm{D}$ band products must be present in wild-type (Y12667) but not in the mutant (D12667) strains. But an A-zeocinB or zeocinC/D band products must be present in the mutant (D12667) strains. (B) Expressed ReCYP51WT and mutants from different yeast. B1 represents the western results of anti-CYP51, B2 represents the western results of anti-xpress (tag protein of plasmid). (C) The growth curve of yeast: D12667 grows slower compared to Y12667 (*p<0.05, D12667 against that of Y12667). While transformed with RePYC/ERG11WT, Re12667WT shows the same growth tendency as Y12667, and PYC/NT/C-ERG11 perfectly complement to the wild type ERG11 phenotype ( $p<0.05$, D12667 against that of Re12667WT). gosterol) for growth. Only when $E R G 11$ mutation was accompanied by inactivation of $E R G 3$ can yeast survive under aerobic conditions. ${ }^{5,18,29)}$ In this study, to dismiss the influence of endogenous ERG11, deletion of ERG11 gene was performed in strain Y12667 carrying defective mutated $E R G 3$ alleles.

With the constructed deletion cassette consisting of two $45 \mathrm{bp}$ segments homologous to the start and stop coding regions of Y12667 ERG11 gene and a Zeocin module selectable marker, a new strain D12667 was produced through the selection of Zeocin resistance $(50 \mu \mathrm{g} / \mathrm{ml})$ and G418 resistance $(100 \mu \mathrm{g} / \mathrm{ml})$. As shown in Fig. 4A, PCR analysis of genomic DNA showed successful deletion of ERG11 gene at the correct locus; consistent with this, Fig. 4B1 showed no CYP51 protein expression in the strain D12667 by Western blot analysis; sterol assay also showed a marked accumulation of lanosterol, indicating the inactivated metabolism function of CYP51 protein (Fig. 5). Next, the wild-type SC5314 ERG11 and mutant ERG11Y118A, ERG11Y118F and ERG11Y118T genes were cloned into low-copy $P Y C / N T / C$ plasmids and introduced into $E R G 3 \Delta / E R G 11 \Delta$ yeast strain D12667, and finally cell lines stably expressing wild type CYP51 and mutations were generated. Introduction of CACYP51 gene into D12667 cells recovered their CYP51 protein expression (Fig. 4B2), almost regained the metabolism function of CYP51 (Fig. 5), and restored their slower growth state (Fig. 4C). The $C$. albicans sterol $14 \alpha$-demethylase in $S$. cerevisiae expression level exceeded $40 \% / \mathrm{mg}$ P450 of microsome protein (data not show).
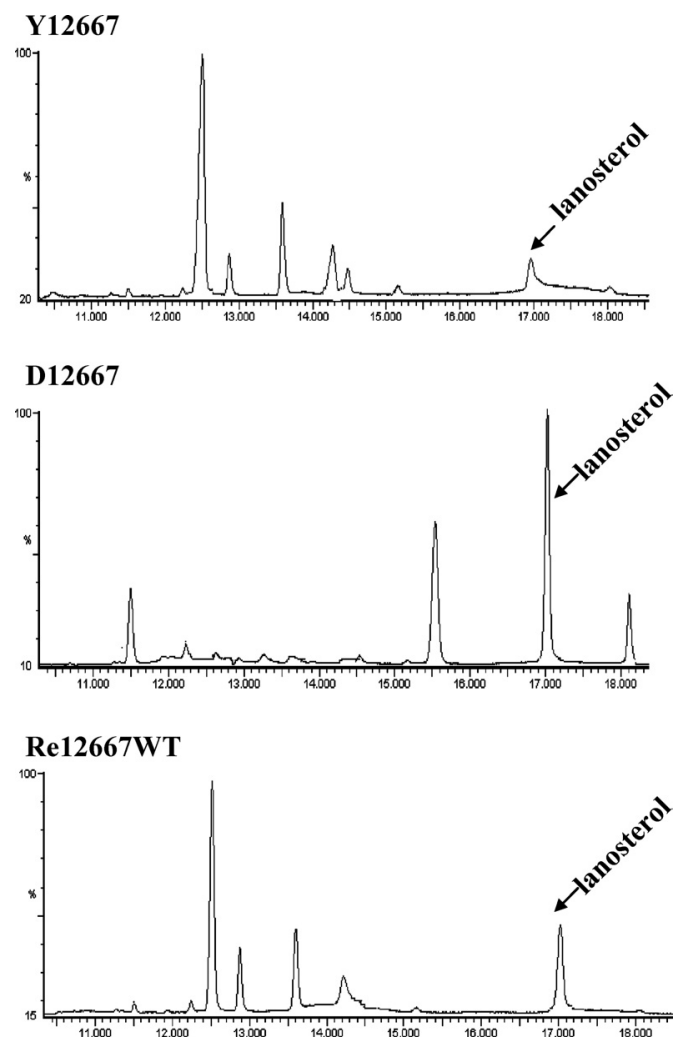

Fig. 5. GC-MS Chromatograms for Levels of Sterol Synthetic Intermediates in Three Yeast Strains

Y12667 harboring a control plasmid accumulated the Ergosta-7,22-dienol; D12667 harboring a control plasmid caused a marked accumulation of lanosterol; Re12667WT complement the wild type ERG11 function with the same sterol accumulation as Y12667. Each sample was measured in triplicates. 
Catalytic Properties of the CYP51 and Variants The catalytic activities of microsomal ReCYP51WT and its mutants were assayed by determining the ability of the enzymes to convert the lanosterol to its $\mathrm{C}-14 \alpha$ demethylated product, 4,4-dimethyl-5 $\alpha$-cholesta-8,14,24-trien-3 $\beta$-ol. The wildtype enzyme CYP51 of $C$. albicans SC5314 expressed in D12667 showed lanosterol $14 \alpha$-demethylase activity about $11.78 \mathrm{nmol} / \mathrm{min} / \mathrm{mg}$ protein upon the reconstruction NADPH recycle system. Introduction of $Y 118 \mathrm{~A}, \mathrm{Y118F}$ and $\mathrm{Y118T}$ substitution into CYP51 of C. albicans SC5314 considerably reduced the catalytic activity of lanosterol $14 \alpha$-demethylase to $6.25,6.54$ and $5.82 \mathrm{nmol} / \mathrm{min} / \mathrm{mg}$ protein respectively (Fig. 6). These $Y 118$ amino acid substitutions seemed to have additive effects on the enzyme activity, since the activity of ReCYP51WT mutants was no more than one-half of that of CYP51WT. These results suggest that Y118 is an important residue for maintaining CACYP51 activity. It was reported ${ }^{2)}$ that a single point mutation in CACYP51 caused unfavorable effects on protein stability and structural conformation, and contributed greatly to enzyme activity and interaction with antifungal inhibitors, which implies Y118 may affect enzyme activity through these pathways. ${ }^{2,30-33)}$ Alternatively, as predicted in our previous modeling method and discussed in our subsequent section, $Y 118$ was recognized as a highly selective residue for the specific interaction with azoles inhibitors and also with natural substrate, implying that the low activity of CACYP51 mutations also might be due to loss of the active enzyme by less binding with substrates.
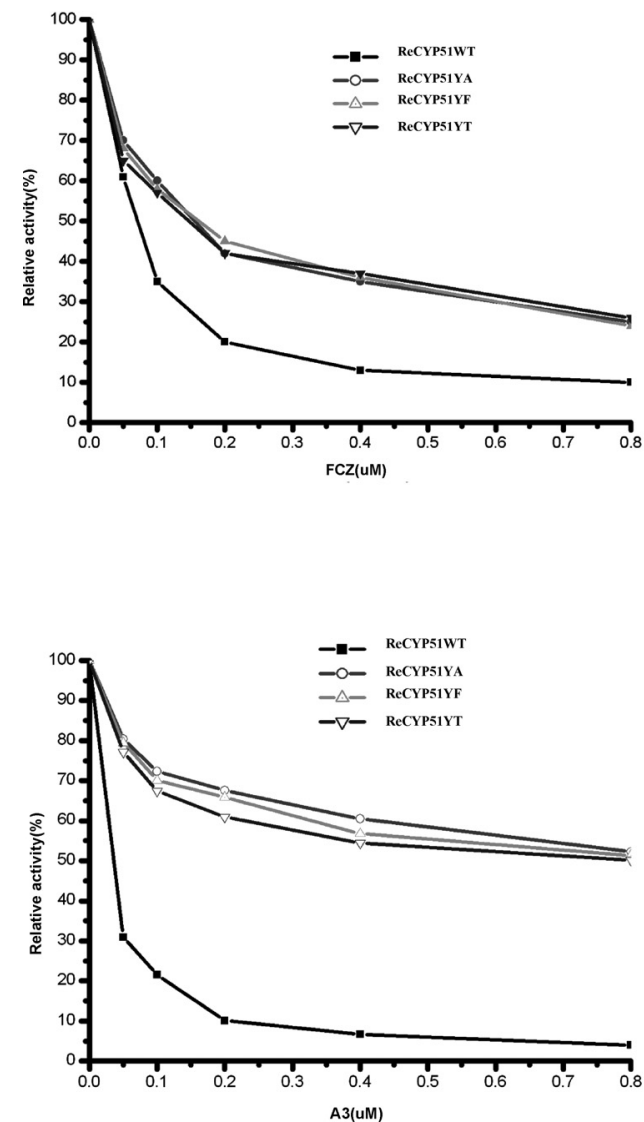

Microsome-Based Assays for the Inhibitory Ability of Azoles Analogous to Recombinant Enzymes Influenced by the $Y 118$ Substitutes On the basis of our proceeding results, a set of novel azoles based on the results from molecular modeling were designed, synthesized and analyzed for greatly improved antifungal activities in in vitro antifungal assay. ${ }^{3-9)}$ The structural modifications of these novel azoles were aimed at the side chains attached to $\mathrm{C} 3$. In the present study, to investigate whether $Y 118$ residue affect interaction of the enzyme with inhibitors, 3 azole derivatives, idiocona-

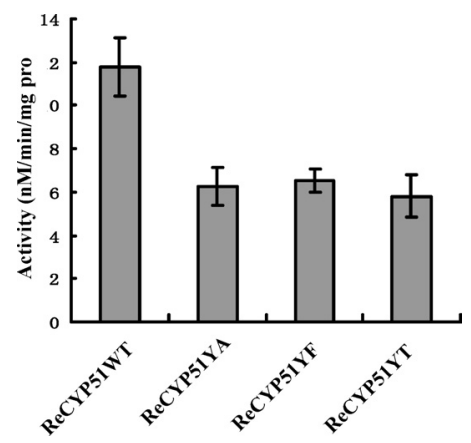

Fig. 6. Lanosterol $14 \alpha$-Demethylase Activities for Microsome Preparations

ReCYP51WT, ReCYP51YA, ReCYP51YF, and ReCYP51YT, respectively prepared from strains of Re12667WT, Re12667YA, Re12667YF and Re12667YT. The lanosterol $14 \alpha$-demethylase activity of ReCYP51WT is statistically higher than those of three other groups $(* p<0.05$, ReCYP51WT against that of three other groups). Each sample was measured in triplicates.
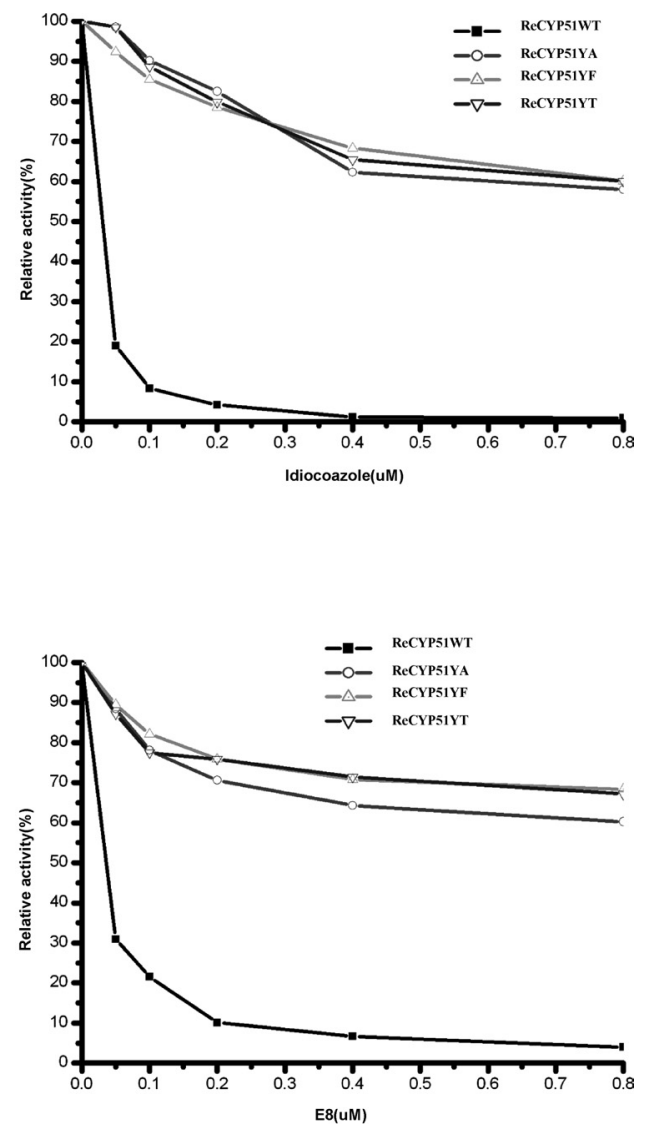

Fig. 7. ReCYP51WT and Variants Influence Antifungal Activities of Azole Derivatives

Lanosterol $14 \alpha$-demethylase activity of microsomes from Re12667WT and variants was determined as described in Materials and Methods. Azole derivatives were added to the reaction mixture at concentration from 0 to $0.8 \mu \mathrm{M}$. 
zole, A3, E8, were selected to further examine the inhibitory ability on the heterologously expressed C. albicans CYP51 for microsomal activity with comparison to fluconazole. The further exploration was based on the varying concentration of the selected azole compounds. A comparative plot of relative activities of microsomal ReCYP51WT and its three variants ReCYP51YA, ReCYP51YF, ReCYP51YT versus varying concentrations of the tested azoles were shown in Fig. 7. The wild-type ReCYP51WT was extremely sensitive to our compounds with apparent $\mathrm{IC}_{50}$ of less than $0.03 \mu \mathrm{M}$ for idiocoazole, $0.04 \mu \mathrm{M}$ for $\mathrm{A} 3$ and $0.035 \mu \mathrm{M}$ for $\mathrm{E} 8$, whereas a $1.5-2$ folds excess of sensitive to fluconazole (FCZ) with apparent $\mathrm{IC}_{50}$ was $0.068 \mu \mathrm{M}$. It is clear that the modification on the $\mathrm{C} 3$ side chain of these azole derivatives was, in a certain, influencing the compounds sensitive to wild type CYP51 enzyme. The $50 \%$ inhibitory concentration $\left(\mathrm{IC}_{50}\right)$ of FLZ to mutation type enzymes was about $0.17-0.20 \mu \mathrm{M}$, about $2.4-2.8$ fold increase when compared to the wild type enzyme. The $\mathrm{IC}_{50}$ of our azole derivatives to mutation type enzymes were increased to $2.4-3.4 \mu \mathrm{M}$, the inhibitory activity decreased about $80-110$ fold when compared to the wild type enzyme. While compared the $\mathrm{IC}_{50}$ of our azole derivatives to mutation enzyme to FLZ, the derivatives exhibited negative inhibitory activity with a $15-20$ fold decrease. These data not only demonstrated that $Y 118$ substitutions occurring in wild type CYP51 enhanced their resistance to our azole derivatives, although this substitution was not found in clinical azole-resistant strain, but also indicated that the lower sensitive effects of the azole derivatives on CYP51 mutates was dependent more on the side chain modification of these derivatives and together on the introduction of mutant to the residue $Y 118$ inducing high resistance of the enzyme to a certain azole compounds.

In the early $1990 \mathrm{~s}$, Heterologous expression of the C. albicans ERG11 gene in $S$. cerevisiae to study the effect of point mutations on which of the amino acid substitutions observed in clinical fluconazole resistant strains conferred a primary effect altering resistance had been produced. Also, some substitutions of CACYP51, such as Y132H, F145L, T315A, $\mathrm{G} 464 \mathrm{~S}$ and $\mathrm{R} 467 \mathrm{~K},{ }^{20,22-26,29)}$ contribute the decrease affinity of azoles to cellular target enzyme have been isolated. The effect of these mutations on the catalytic activity and azole resistance has been discussed in detail. However, these substitutions are mainly from resistant clinical isolates of Candida albicans and the aim of these studies is focused on the importance of these residues on azole resistance. In the present study, we select $Y 118$ for mutation because it can form important $\pi-\pi$ stacking interaction with the highly active antifungal compounds according to our modeling results. The elucidation of the importance of $Y 118$ can not only explain its role in catalytic activity and azole sensitivity, but also can identify an important drug binding site for the rational design of novel CACYP51 inhibitors with potent antifungal activity.

\section{CONCLUSION}

In our previous studies, a 3D model of CACYP51 enzyme was established, in which the quantitative structure-activity relationship method and molecular docking were used to investigate enzyme-inhibitor interactions using a set of novel azole antifungal compounds, ${ }^{3-7)}$ and demonstrated that sev- eral important residues interacted with azole derivatives in the active site of CACYP51, among which Y118 could form $\pi-\pi$ interaction with the phenyl group side chain attached to $\mathrm{C} 3$ of azole derivatives. These findings predicted a hypothesis that $Y 118$ may be an important residue for the binding of CACYP51 inhibitors through the phenyl group side chain. Therefore, the present study is to further verify the prediction and reliability of above described model. Specifically, sitedirected mutagenesis of the $Y 118$ residue of $C$. albicans CYP51 heterologously expressed in $S$. cerevisiae was undertaken with deletion of the ERG11 gene. Alteration of $Y 118$ with $A, F, T$ by a single base substitution produced an altered protein exhibiting reduced metabolic activity to natural substrate lanosterol, which suggested that Y118 residue may be essential for CACYP51 activity in maintaining heme environment. Furthermore, our designed antifungal compounds showed more potent activities on wild type CACYP51 and less on mutant ones than fluconazole. Consistently, the enzyme inhibition results also demonstrated that they had better effects on cells expressing wild type CYP51 proteins in MICs assay. These findings implied that the role of residue Y118 in interacting with the special structure of our synthesized compounds, which further validated the reliability of previously established molecular modeling results. Taken together, $Y 118$ was demonstrated to be essential for maintaining CACYP51 activity and important for interaction with special phenyl group side chain attached to $\mathrm{C} 3$ of azole derivatives, which may further guide the rational design of CACYP51 inhibitors.

Acknowledgments This study is supported by the National Natural Science Foundation of China (Grant No. 30430750).

\section{REFERENCES}

1) Lupetti A., Danesi R., Campa M., Tacca M. D., Kelly S., Trends Mol. Med., 8, 76-81 (2002)

2) Lepesheva G. I., Virus C., Waterman M. R., Biochemistry, 42, 90919101 (2003).

3) Sheng C. Q., Zhang W. N., Ji H. T., Zhang M., Song Y. L., Xu H., Zhu J., Miao Z. Y., Yao J. H., Zhou Y. J., Lu J. G., J. Med. Chem., 46, 474 485 (2005).

4) Ji H., Zhang W., Zhou Y., Zhang M., Zhu J., Song Y., Lu J., J. Med. Chem., 43, 2493-2505 (2000).

5) Lewis D. F., Wiseman A., Tarbit M. H., J. Enzyme Inhib., 14, 175192 (1999).

6) Li X., Madison V., Chau A. S., Loebenberg D., Palermo R. E., McNicholas P. M., Antimicrob. Agents Chemother, 48, 568-574 (2004).

7) Fukuoka T., Johnston D. A., Winslow C. A., Antimicrob. Agents Chemother, 47, 1213-1219 (2003).

8) Sheng C. Q., Zhu J., Zhang W. N., Song Y. L., Zhang M., Ji H. T., Yu J. X., Yao J. Z., Yang S., Miao Z. Y., Yaoxue Xue Bao, 39, 984-989 (2004).

9) Sheng C., Zhang W., Zhang M., Song Y., Ji H., Zhu J., Yao J., Yu J., Yang S., Zhou Y., Zhu J., Lu J., J. Biomol. Struct. Dyn., 22, 91-99 (2004).

10) Ji H., Zhang W., Zhang M., Kudo M., Aoyama Y., Yoshida Y., Sheng C., Song Y., Yang S., Zhou Y., Lu J., Zhu J., J. Med. Chem., 46, 474 485 (2003)

11) Lepesheva G. I., Podust L. M., Bellamine A., Waterman M. R., J. Biol. Chem., 276, 28413-28420 (2001).

12) Bellamine A., Mangla A. T., Dennis A. L., Nes W. D., Waterman M. R., J. Lipid Res., 42, 128-136 (2001).

13) Podust L. M., Poulos T. L., Waterman M. R., Proc. Natl. Acad. Sci. U.S.A., 98, 3068-3073 (2001). 
14) Sanglard D., Kuchler K., Ischer F., Pagani J. L., Monod M., Bille J., Antimicrob. Agents Chemother, 39, 2378-2386 (1995).

15) Sanglard D., Ischer F. F., Koymans L., Bille J., Antimicrob. Agents Chemother, 42, 241-245 (1998).

16) Kelly S. L., Lamb D. C., Kelly D. E., FEMS Microbiol. Lett., 180, $171-175$ (1999).

17) White T. C., Antimicrob. Agents Chemother, 41, 1488-1494 (1997).

18) Geber A., Hitchcock C. A., Swartz J. E., Pullen F. S., Marsden K. E., Kwon-Chung K. J., Bennett J. E., Antimicrob. Agents Chemother, 39, 2708-2717 (1995).

19) Francisco C. H., Maryse T., Nathalie R., Kahn S. B., Alain R., Danieále W. R., Eur. J. Biochem., 262, 435-446 (1999).

20) Lamb D. C., J. Biol. Chem., 272, 5682-5688 (1997).

21) Matsuura K., Yoshioka S., Tosha T., Hori H., Ishimori K., Kitagawa T., Morishima I., Kagawa N., Waterman M. R., J. Biol. Chem., 280, 9088-9096 (2005).

22) Asai K., Tsuchimori N., Okonogi K., Perfect J. R., Gotoh O., Yoshida Y., Antimicrob. Agents Chemother, 43, 1163-1169 (1999).

23) Lamb D. C., Kelly D. E., White T. C., Kelly S. L., Antimicrob. Agents Chemother, 44, 63-67 (2000).

24) Favre B., Didmon M., Ryder N. S., Microbiology, 145, 2715-2725
(1999).

25) Kayeya H., Inoney Sawai Y., Ikuta Y., Ohno Y., Antimicrob. Agents Chemother, 44, 2985-2990 (2000).

26) Chiatogu O., Jill R. B., Maurizio D. P., Joseph H., Antimicrob. Agents Chemother, 47, 956-964 (2003).

27) Brachmann C. B., Davies A., Cost G. J., Caputo E., Li J., Hieter P., Boeke J., Yeast, 14, 115-132 (1998).

28) Alloush H. M., Edwards T. A., Lisboa V. V., Wheals A. E., Yeast, 19, $79-86$ (2002).

29) Sanglard D., Ischer F. O., Falconer D., Bille J., Antimicrob. Agents Chemother., 47, 2404-2412 (2003).

30) Bellamine A., Mangla A. T., Nes W. D., Waterman M. R., Proc. Natl. Acad. Sci. U.S.A., 96, 8937-8942 (1999).

31) Jackson C. J., Lamb D. C., Marczylo T. H., Parker J. E., Manning N. L., Kelly D. E., Kelly S. L., Biochem. Biophys. Res. Commun., 301, $558-563$ (2003).

32) Aoki Y., Yoshihara F., Kondoh M., Nakamura Y., Nakayama N., Arisawa M., Antimicrob. Agents Chemother, 37, 2662-2667 (1993).

33) Matsumoto M., Ishida K., Konagai A., Maebashi K., Asaoka T., Antimicrob. Agents Chemother., 46, 308-314 (2002). 